\title{
Spontaneous Gallbladder Perforation: A case series
}

\author{
Sujit Kumar, Sanjay Negi , B.N Patowary, Aditya Jalan, Sulabh Rajbhandari \\ Department of Surgery, College of Medical Sciences-Teaching hospital \\ Bharatpur, Chitwan , Nepal \\ Correspondence : Dr Sujit Kumar, Associate professor, Department of Surgery, College of Medical sciences- \\ Teaching Hospital ,Bharatpur, Chitwan, Nepal \\ Email-drsujit1755@gmail.com
}

\begin{abstract}
Background: Gallbladder is rarely injured as it is embedded in the liver and well protected by ribcage. Gallbladder perforation is a rare complication of acute calculus cholecystitis. Spontaneous gallbladder perforation is even rarer.
\end{abstract}

Case Series: We present our experience of 4 cases of spontaneous gallbladder perforation. The patients were mostly males (3:1) with age ranging from 42-64 years. All the patients presented with abdominal pain, distension, ileus and fever ranging from 2-5 days duration. There was no prior history of biliary colic. Examination revealed abdominal tenderness and ileus. Abdominal radiographs showed multiple air-fluid levels and no pneumoperitoneum. Sonography and CT scan of abdomen revealed pericholecystic fluid, intra-abdominal collection with no evidence of gallstones.

Provisional diagnosis was peritonitis in 3 and acute pancreatitis in one case. Image guided abdominal paracentesis yielded bilious fluid. In view of lack of clinical improvement and aspiration of bilious content all the patients were subjected to laparotomy. Gallbladder perforation in fundus region without stones and intra-abdominal bile collection was noted in all the patients. Cholecystectomy with peritoneal lavage and abdominal drainage was performed in all cases. Postoperative course was uneventful except wound infection in 2 cases. Histopathology report revealed features of acute cholecystitis in 2 and acute on chronic cholecystitis in the remaining 2 patients.

Conclusion: Diagnosis of spontaneous gallbladder perforation should be suspected in middle aged patients who present with acute abdomen but does not have classical signs of peritonitis and in whom paracentesis has yielded bilious fluid.

Keywords: Gallbladder perforation, peritonitis, spontaneous perforation 